\title{
Estimaciones de la migración mexicana de retorno y la necesidad de políticas y programas para su atención
}

\author{
Graciela Martínez Caballero ${ }^{1^{*}}$ y Mónica Martínez de la Peña ${ }^{1^{*}}$ \\ Centro de Estudios Migratorios de la Unidad de Política Migratoria \\ Unidad de Política Migratoria, Registro e Identidad de Personas, \\ de la Secretaría de Gobernación
}

\begin{abstract}
Resumen
El documento presenta un marco de referencia de los conceptos asociados a la migración de retorno y los instrumentos que captan información al respecto, con la finalidad de identificar tendencias y características de la población migrante mexicana que retorna, principalmente desde Estados Unidos. Dicho marco es el punto de partida para la estimación de la migración mexicana de retorno en los periodos 1990-2000 y 2010-2015, así como de los retos para su medición y algunas propuestas para el diseño de políticas públicas en la materia.
\end{abstract}

Palabras clave: migración de retorno, política pública, medición.

\section{Estimates of Mexican return migration and the need for Public Policies} for its attention

\section{Abstract}

This paper presents a framework for concepts associated with return migration and the data source that capture information in this use, in order to identify trends and characteristics of the returning Mexican migrant population, mainly from the United States. This framework is the starting for estimating Mexican return migration in the periods 1990-2000 and 2010-2015, as well as the challenges to their measurement and some proposals for the design of public policies in this area.

Keywords: return migration, public policies, measurement.

1 Las autoras agradecen el procesamiento de algunas cifras a Nicéforo Delgadillo Aguilar, Luis Felipe Ramos Martínez y Eduardo Granados García, colega y ex colegas, respectivamente, de la Unidad de Política Migratoria, Registro e Identidad de Personas (UPMRIP).

* Colaboradoras de la UPMRIP. Las autoras son responsables de las opiniones y reflexiones vertidas en este documento y no reflejan, necesariamente, la visión institucional de la UPMRIP.

Artículo recibido el 06 de mayo de 2019 y aprobado el 27 de agosto de 2019. 


\section{INTRODUCCIÓN}

$\mathrm{E}$

$n$ México convergen con gran dinamismo las cuatro dimensiones de la migración (emigración, inmigración, tránsito y retorno) ya que el corredor fronterizo entre los países de Centroamérica, México y Estados Unidos es uno de los más transitados del mundo y está conformado por migrantes con perfiles y motivaciones distintas, lo que hace de éste un escenario de gran complejidad.

En el siglo pasado, el auge de la emigración de población mexicana hacia Estados Unidos obedeció a la falta de oportunidades de desarrollo en el lugar de origen y al mejoramiento de las condiciones de vida, lo que se conoce como migración por razones económicas; de hecho, en su momento hubo programas bilaterales - como el llamado Programa Bracero (1942-1964) - que incentivaron esta emigración ya que beneficiaba a ambos países.

Cabe mencionar que parte de este auge guarda relación con el crecimiento de la migración no documentada, sobre todo en las últimas décadas del siglo pasado, tras el agotamiento de las prácticas de atracción de migrantes hacia Estados Unidos para desarrollar trabajos vinculados a proyectos específicos de largo alcance. Fue entonces cuando la política migratoria estadunidense se fue tornando hacia esquemas de control fronterizo cada vez más rigurosos. A lo largo del presente siglo, el endurecimiento de la política estadounidense ha dado lugar a la migración de retorno de nuestros connacionales.

En este documento se presenta un marco de referencia para este tema de estudio con base en el análisis de los conceptos asociados a la migración de retorno, así mismo, se establecen las principales precisiones en los instrumentos de captación que se utilizan para estimar el retorno (censos, encuestas y estadísticas construidas a partir de registros administrativos), a efecto de identificar tendencias y características de la población migrante mexicana que retorna, principalmente desde Estados Unidos de América.

Posteriormente, planteamos nuestra postura respecto de la necesidad de contar con una política real de integración a la sociedad mexicana para quienes regresan al país de forma voluntaria o involuntaria como parte de la migración de retorno. En este sentido, 
manifestamos la preocupación por la ausencia de una política efectiva en esta materia, aunque se reconocen algunos esfuerzos que de manera aislada se han llevado a cabo en este sentido.

A manera de contexto, se presenta en esa misma sección un breve recuento de la política migratoria estadounidense como el escenario donde transcurren los principales eventos de repatriación de población mexicana y las hipótesis de impacto que se han generado respecto del retorno, con énfasis en la migración calificada como uno de los grandes rubros a considerar en la política social de integración, por su potencial y por los beneficios que puede traer su inserción laboral en las economías locales.

Finalmente, se presentan estimaciones de la migración mexicana de retorno en los periodos 1990-2000 y 2010-2015, las características sociodemográficas de esta población, así como algunas propuestas para el diseño de políticas públicas en la materia.

\section{MARCo DE REFERENCIA PARA EL ANÁLISIS DE LA MIGRACIóN DE RETORNO}

\section{¿De qué hablamos cuando referimos el retorno?}

El análisis de un fenómeno tan dinámico como el desplazamiento de las poblaciones a menudo impone retos conceptuales que contribuyan a explicar su dinamismo. El estudio de la migración internacional, sus enfoques y dimensiones, es uno de los más complejos ya que además de los factores inherentes como la temporalidad o la distancia, los motivos en los que descansa el desplazamiento son cada vez más diversos. Sin embargo, para efectos de medición o estimaciones es indispensable delimitar conceptualmente el fenómeno de estudio para contar con aproximaciones adecuadas.

Técnicamente, la migración de retorno alude a aquellas personas que nacieron y vivieron en México una parte de su vida, pero emigraron hacia otro país para residir allá por un tiempo determinado por causas voluntarias o involuntarias y, de la misma manera, regresaron a México.

En este documento se retoma el concepto de la Organización Internacional para las Migraciones (OIM), quien define a la migración de retorno como el "movimiento de personas que regresan a su país de origen o a su residencia habitual, generalmente después de 
haber pasado por lo menos un año en otro país. El retorno puede ser voluntario o no. Incluye la repatriación voluntaria" (OIM, 2006: 39).

Cabe mencionar la diferencia entre el retorno voluntario e involuntario. Siguiendo a la OIM, el retorno voluntario es definido como "el regreso independiente o asistido al país de origen, de tránsito o a un tercer país basado en la libre voluntad de la persona que regresa" (OIM 2006: 65). Debido a que ésta es una definición muy amplia dentro de la que pudieran entrar varios programas operados para extranjeros en situación irregular que son devueltos por una autoridad migratoria, para los fines de este documento, nos referimos al retorno voluntario como el que se realiza sin mediación de autoridad migratoria alguna, independientemente de los factores o causas que llevaron a la persona migrante a regresar a su país de origen.

Por su parte, el retorno de personas migrantes (generalmente en situación de irregularidad) donde media una autoridad migratoria es entendido en este trabajo como retorno involuntario.

Es importante aclarar que no se utiliza el concepto de retorno forzado o migración forzada, ya que se ha identificado en la literatura que este concepto es utilizado, principalmente para referir los desplazamientos de poblaciones con necesidad de protección internacional (refugiada); o en el marco de las llamadas crisis humanitarias y que implica múltiples factores que van desde el desplazamiento por cambio climático, violencia generalizada, o violación a derechos humanos, hay incluso quienes lo utilizan para referir la migración forzada por motivos económicos, aun cuando fue una decisión personal. ${ }^{1}$

Otro elemento que generalmente se considera para precisar la dimensión del retorno es el núcleo familiar que regresa con el migrante, principalmente hijas e hijos que nacieron en el país de destino, esto es importante para efectos de programas de atención y políticas de integración, sin embargo, toda vez que en sentido estricto no forman parte del flujo de retorno porque nunca salieron del país de origen de los padres, no son considerados en las estimaciones que más adelante se presentan.

Además de lo anterior es importante señalar que, si bien en términos conceptuales se cuenta con una definición de la migración de retorno y se han realizado adecuaciones a las referencias sobre su identificación, en la práctica, la cuantificación no sólo de esta

1 Véase Martin, Susan, Sanjula Weerasinghe y Abbie Taylor (2014). "What is crisis migration" http://www.fmreview.org/crisis/martin-weerasinghe-taylor\%20.html 
dimensión de la movilidad internacional sino de cualquier otra está supeditada a la disponibilidad de la información para medirla y a la definición que de ésta se haga con los instrumentos de captación, tema que se aborda en el siguiente apartado.

\section{CRITERIOS PARA LA MEdición DE LA Migración DE RETORNo}

En los censos y encuestas de población en México, se considera que un migrante de retorno es aquel migrante internacional que al momento de la entrevista se encontraba residiendo nuevamente en México, en tanto la identificación de un migrante internacional se basa en la salida del país al menos una vez, ya sea con la intención de vivir, estudiar o trabajar en el extranjero durante los últimos cinco años, partiendo de la fecha de la entrevista (INEGI, 2014-2015), es decir, no se circunscribe el retorno a la estancia mínima de un año, lo que permite que en esta amplitud de tiempo se mezcle a los migrantes circulares.

Ante este reto conceptual y metodológico de la captación de información, en un trabajo conjunto, la Unidad de Política Migratoria y el Consejo Nacional de Población, realizaron definiciones operativas que permitieran identificar a los migrantes de retorno considerando los criterios que se mencionan a continuación. A partir de registros administrativos y de la Encuesta sobre Migración en la Frontera Norte de México y algunas consideraciones de carácter normativo que permiten operar el concepto de retorno de la siguiente manera:

a) La temporalidad de estancia en el país de destino (básicamente Estados Unidos de América, considerando la factibilidad de haber logrado el inicio de una intención de residencia);

b) La temporalidad de permanencia en México, y

c) Las veces que fue devuelto por una autoridad migratoria a México.

Temporalidad de estancia en el país de destino. Aunque la recomendación de la OIM es considerar mínimo un año de estancia en el país de destino, antes de retornar, se ha considerado que con más de seis meses de estancia en otro país las personas migrantes iniciaron una perspectiva de vida con uno o varios empleos, principalmente aquellos que cuentan con redes de apoyo. Por otro lado, estimaciones realizadas a partir de la Encuesta Nacional de la Dinámica Demográfica 2014, muestran que entre los mexicanos que ingresaron a Estados Unidos de América con visa de turista para una estancia regular de 
máximo seis meses, el 41.1 por ciento permanece más tiempo del autorizado (la mayoría permanece entre siete meses a dos años).

La temporalidad de permanencia en México. Si bien el tiempo de estancia en el país de origen no es un elemento de la conceptualización de la migración de retorno, es importante considerar esta dimensión en el diseño de acciones dirigidas a una política pública en esta materia ya que muchos migrantes intentan regresar a Estados Unidos de América una o varias ocasiones en el mismo año, lo que lleva a una duplicidad de eventos que forman parte de otro tipo de políticas.

Número de veces que fue devuelto por una autoridad migratoria a México. Este criterio está muy vinculado al anterior y está relacionado directamente al flujo de retorno involuntario irregular. Es importante diferenciar en primera instancia que los números globales hacen referencia a eventos, es decir, registran el número de veces que fue devuelta una misma persona en el lapso de un año. En este sentido, es importante establecer con la mayor precisión posible cuántas personas son las que integran los flujos de retorno, ya que quienes intentan cruzar varias veces claramente no quieren quedarse en México.

Además, de las consideraciones anteriores para identificar a las personas de retorno en el diseño de una política pública, es importante determinar las particularidades de los flujos migratorios, ya que la precisión de su objetivo y el éxito en su ejecución depende en cierta medida de la identificación de la población objetivo, es decir, a quien está dirigida.

En el caso de las y los migrantes mexicanos de retorno, es importante definir si se requiere una política pública de atención y reintegración que considere el tiempo de residencia en Estados Unidos de América, ya que probablemente estos migrantes se encontrarán con un contexto social, político y económico distinto al que se vivía cuando se fueron y a su regreso no cuenten con redes de apoyo y, no tengan un lugar donde vivir ya que han perdido cualquier vínculo con México, es decir, para esta población se requiere de una política pública con perspectiva de reintegración social que facilite un desarrollo óptimo y bienestar.

En contraste, si se desea establecer una política de retención, se tendrá que incluir a la población con múltiples intentos de cruce hacia Estados Unidos de América con la finalidad de ofrecer alternativas a la migración, política que necesariamente tendrá que considerar a toda la población mexicana si lo que se quiere es evitar la experiencia 
migratoria, es decir, se tendrá que implementar una política pública con perspectiva de prevención.

Para cuantificar este universo poblacional del que se habla no se puede hacer referencia a los eventos migratorios per se sino al número de intentos de cruce que realizan las personas migrantes a partir del número de devoluciones de las que fueron objeto por la autoridad migratoria estadunidense.

Con estas consideraciones se conformaron definiciones intermedias del retorno según las fuentes de información disponibles:

Censos y Encuesta Intercensal

Persona que nació en México y que hace cinco años vivía en otro país y, al momento de la entrevista se encontraba residiendo nuevamente en México.

Dichas fuentes hacen referencia a personas.

Encuesta sobre Migración en la Frontera Norte de México (Emif Norte)

Persona que nació en México y que residió o permaneció más de seis meses en Estados Unidos de América y se encuentra en México con la intención de permanecer en el país al menos un año.

La fuente capta eventos, pero los criterios aplicados permiten hacer una equiparación a personas para referir el retorno voluntario con frecuencia anual.

Estadísticas Migratorias

Persona que nació en México y que residió o permaneció más de seis meses en Estados Unidos de América y que fue devuelta a México en una sola ocasión por las autoridades migratorias norteamericanas. La fuente capta eventos, no obstante, a través de un proceso de identificación se ha logrado identificar personas, lo que junto con los criterios aplicados permiten estimar el retorno involuntario.

\section{Metodología}

En México, no hay una sola fuente de información que dé cuenta de las tendencias de la migración de retorno de manera periódica, por lo que es necesario complementar con fuentes de información que nos permitan acercarnos a la tendencia del fenómeno y las características de las poblaciones, ya que en un ejercicio ideal de diseño de política en la materia deberían de considerarse todos los elementos posibles.

Por lo anterior, en este trabajo abordamos de manera diferenciada la información disponible para el retorno de población mexicana migrante. En lo que respecta al censo y la encuesta intercensal, la 
cifra da cuenta de quienes regresaron en el quinquenio, pero no de forma anual, excepto por la estimación del promedio; sin embargo, nos permite identificar características sociodemográficas de esta población y su situación al momento de la encuesta, así como deducir a partir de los motivos de regreso si éste fue voluntario o involuntario.

Por otro lado, tanto la Encuesta sobre Migración en la Frontera Norte (EMIF) como la estadística derivada del registro administrativo, nos permiten estimar el volumen anual de personas que retornan a México desde Estados Unidos de América de forma voluntaria e involuntaria a partir de preguntas como el tiempo de estancia en ese país, la intención de permanencia en México (no pensaban regresar a Estados Unidos de América), y a quienes fueron identificados con un solo evento de repatriación en el año.

No obstante, la riqueza de la información, la EMIF sólo capta a las poblaciones de 15 años o más. Para estimar a este grupo se utilizó la estructura por edad a partir de la Encuesta Nacional de la Dinámica Demográfica (ENADID) para obtener la información de 2014 y 2015. Otro aspecto que debe considerarse al leer los datos es que la estimación incluye sólo a quienes se encontraban en Estados Unidos de América, pues tanto la EMIF como la estadística migratoria solo permiten identificar dicho origen. Aunque ello podría considerarse una limitación, cabe recordar que alrededor del 95 por ciento de nuestros connacionales se encuentran en ese país.

La metodología utilizada es sólo una aproximación al retorno de mexicanos que debe aún ser perfeccionada para incluir otro tipo de información como el de visas emitidas por otros países, lo que podría contribuir a llenar uno de los vacíos de la información para el caso del retorno voluntario, en tanto no se tenga un mecanismo que permita identificar dichos movimientos.

En esencia, la estimación no solo busca dar cuenta de las tendencias anuales, sino de analizarlas bajo las múltiples implicaciones que tienen desde la perspectiva de la política pública, que van no sólo desde las acciones que deben tenerse en consideración para la reintegración de estas personas en la sociedad mexicana, sino de ubicar el fenómeno en su dimensión y no dejarse llevar por especulaciones de retornos masivos como consecuencia de la política migratoria estadounidense. 


\section{LA MIGRACIÓN MEXICANA DE RETORNO Y LA NECESIDAD DE POLÍTICAS INTEGRALES PARA SU ATENCIÓN}

La comunidad mexicana residente en Estados Unidos de América en condición regular o irregular - es decir, con o sin documentos que acrediten una estancia legal en ese país- era alrededor de 11.5 millones de personas en 2017, lo que significa el establecimiento de familias enteras de origen mexicano con o sin vínculos en nuestro país. De éstos, se estima que alrededor de 5.6 millones son inmigrantes en situación migratoria irregular expuestos a las políticas de deportación.

La gestión del ex presidente estadounidense Barack Obama-pese a su diplomático discurso a favor de una reforma migratoria y del impulso que dio a las acciones diferidas para una eventual regularización de población migrante- se caracterizó por un volumen importante de deportaciones de población mexicana: más de 2.7 millones de eventos entre 2009 y 2015, superando las ocurridas en el periodo presidencial de George W. Bush cuando se deportó a dos millones de personas entre 2001 y 2008 (Department of Homeland Security, 2015).

La inercia de la política establecida en 2007 ha sido desde entonces el escenario para crear expectativas de retorno masivo de connacionales a nuestro país, cuyo eje han sido las deportaciones desde el interior de Estados Unidos de América, el reforzamiento de la frontera con agentes migratorios y la tecnología implementada en las acciones de detección.

En este sentido, mucho se ha especulado acerca del retorno masivo de migrantes hacia México durante la presidencia del actual mandatario Donald Trump y su propuesta de una reforma migratoria centrada en la seguridad nacional, bajo la idea de que la migración ilegal es una amenaza para Estados Unidos de América y la construcción de un muro fronterizo. Como parte de estas medidas también destaca el reforzamiento a la ley migratoria para detener y deportar personas migrantes desde el interior del país (The White House, 2017).

A la luz de lo anterior, en 2018 se registró un incremento del 21.9 por ciento de los eventos de devolución de mexicanos respecto de 2017, cifra que está muy alejada de las devoluciones recibidas por el INM en 2009 (602 mil eventos).

Lo que en realidad nos preocupa —además de las cifras- es la ausencia de una política de integración de esta población de retorno a la sociedad mexicana, pensada en términos de su inclusión efectiva 
mediante el pleno ejercicio de los derechos humanos. En esta lógica deben considerarse muchos elementos no sólo en relación con el número de personas devueltas por las autoridades migratorias, o que regresan de forma voluntaria, sino el perfil sociodemográfico y sus necesidades específicas, así como los vínculos familiares que se tienen de cara a la reunificación familiar.

La gran deuda del Estado mexicano en esta materia ha sido la ausencia de una política migratoria de retorno asertiva, se han hecho algunos intentos incipientes para atender momentáneamente a nuestros connacionales que regresaron al país de forma involuntaria por medio de una deportación, pero no se aspiró a más de la atención a la salud de primer contacto y la inscripción a bolsas de trabajo para algún programa de empleo en ciudades específicas, además de que no hubo un seguimiento de estas acciones y mucho menos una política real de integración.

Destacan también los esfuerzos en materia educativa para facilitar la inserción escolar de estudiantes en los flujos de retorno al sistema nacional, sin embargo, uno de los grandes obstáculos de esta iniciativa que mucho prometía tiene que ver con la incompatibilidad de las políticas entre los tres órdenes de gobierno. En particular, esta iniciativa a nivel nacional no prosperó en todas las comunidades de acogida de migrantes de retorno.

Desde la perspectiva de la reintegración, debe considerarse a todas y todos nuestros connacionales que regresan al país con independencia del carácter de su retorno, voluntario o involuntario, e incentivando así mismo las condiciones óptimas para su permanencia en nuestro país a efecto de que la migración no sea una necesidad. Es importante destacar que el retorno voluntario puede enmascarar una determinación personal respecto de este evento, es decir, el hecho de que el retorno no sea producto de una deportación no necesariamente significa que sea un acto estrictamente voluntario, sino que puede ser motivado por las presiones de la política estadounidense y de las medidas puestas en marcha en el marco del exacerbado control migratorio, además del clima hostil antiinmigrante gestado en una parte de la sociedad norteamericana.

Otro aspecto que definitivamente juega un rol preponderante en los procesos de retorno es la reunificación familiar, en este sentido, el Estado mexicano tiene un reto mayor a efecto de favorecer este 
proceso con apego al respeto irrestricto de los derechos humanos y privilegiando el interés superior de la niñez, cuando sea el caso.

En el ideal de una política incluyente debe tomarse en cuenta la diversidad de la población de retorno y sus necesidades específicas según características sociodemográficas. Por ejemplo, en el caso de niñas, niños y adolescentes (NNA) hay que tener en cuenta el dominio del idioma español ya que es un aspecto clave para su integración en la sociedad mexicana, así como la homologación de los planes de estudio en el sistema educativo nacional.

Uno de los grupos que se analiza en este documento son las y los migrantes calificados; esto, por su gran potencial como agentes de cambio en el mercado laboral y por los retos que implica el adecuado aprovechamiento de este potencial para ambas partes: fuerza de trabajo y mercado laboral. Consideramos que se trata de migración calificada en función del nivel de estudio, uno de los criterios más utilizados para medirla, aunque existen otros como las habilidades extraordinarias adquiridas, la experiencia laboral o los puestos de trabajo desempeñados.

En complemento, otra de las propuestas que planteamos en este documento se refiere a la perspectiva de políticas de prevención del fenómeno emigratorio, es decir, aspirar a que el evento migratorio sea una decisión más que una necesidad. Se trata de políticas que contribuyan al desarrollo económico y el bienestar de las personas en todas las regiones de nuestro país a efecto de mitigar los motivos que llevan a la necesidad de emigrar.

\section{ESTIMACIONES DE LA MIGRACIÓN DE RETORNO A MÉXICO}

A partir de los criterios establecidos en este documento se estimó la migración de retorno a partir de las fuentes disponibles, tanto de censos y encuestas generadas por el INEGI, como de los registros administrativos que permiten dar seguimiento a este fenómeno de manera anual.

Según estimaciones realizadas con la Encuesta Intercensal 2015, en el quinquenio 2010-2015 retornaron a México 495 mil 434 mexicanos, de los cuales, 89 por ciento provenían de Estados Unidos de América (véase Tabla 1). Si se asume que la tendencia se mantuvo igual en todos los años podría decirse que, en promedio, 99 mil mexicanos retornaron cada año en ese periodo, independientemente del año en 
el que hayan emigrado o el tiempo de permanencia en Estados Unidos de América.

Tabla 1. Estimación de la migración de retorno quinquenal, a partir de la Encuesta Intercensal, 2015

\begin{tabular}{crrr}
\hline Fuente & \multicolumn{3}{c}{ Lugar de residencia hace cinco años } \\
\hline Total & $\begin{array}{c}\text { Estados Unidos } \\
\text { de América }\end{array}$ & Otro país \\
Encuesta & 495,434 & 442,503 & 52,931 \\
Intercensal & $(100.0 \%)$ & $(89.3 \%)$ & $(10.7 \%)$ \\
\hline
\end{tabular}

Fuente: Estimaciones de la Unidad de Política Migratoria y del Consejo Nacional de Población de la SEGOB con base en INEGI, Encuesta Intercensal, 2015; e INEGI, Censo de Población y Vivienda, 2010, muestra del 10 por ciento.

Del total de migrantes que regresaron de Estados Unidos de América en 2015, 9.8 por ciento residía en Jalisco, 7.8 por ciento en Michoacán y 7.0 por ciento en Baja California. Por su parte, 12.3 por ciento de las personas que retornaron de otro país residía en la Ciudad de México, 9.3 por ciento en el Estado de México y 9.1 por ciento en Jalisco (véase Figura 1).

Con la finalidad de obtener estimaciones anuales, a partir de la Encuesta sobre Migración en la Frontera Norte de México (Emif Norte) y de las estadísticas migratorias obtenidas de registros administrativos, realizamos otras estimaciones considerando diferentes tiempos de estancia en Estados Unidos de América y de permanencia en México, según los criterios descritos con anterioridad. La primera estimación asume seis meses de estancia en el país del norte y seis meses de permanencia en México, cuyo volumen de retorno va de 211 mil 189 personas a 176 mil 519 entre 2014 y 2016. Es necesario precisar que estas cifras deben tomarse con cautela ya que debido a las temporalidades utilizadas se mezclan eventos y personas, es decir, la cifra considera las internaciones a México en más de una ocasión, lo que pone en duda la intención de permanencia en nuestro país (véase Tabla 2).

A diferencia de los dos primeros escenarios, en el tercero se estima el número de personas de retorno en sentido estricto, es decir, aplica para aquellas personas que manifestaron haber permanecido un año en Estados Unidos y un año en México, o bien, que no piensan regresar al vecino país del norte. Por la importancia que representa para la política nacional el conocer la magnitud de este fenómeno 
con la mayor precisión posible para la planeación y programación correspondientes, en este escenario la estimación de la permanencia en el país adquiere un mayor peso, por ello se considera que quienes no tenían una estancia lo suficientemente consolidada en el país de destino y no intentaron un nuevo cruce tienen una mayor probabilidad de quedarse en México.

Figura 1. Estimaciones de la migración de retorno quinquenal, según estado de residencia y país de procedencia, 2015

Retornaron de EE. UU.

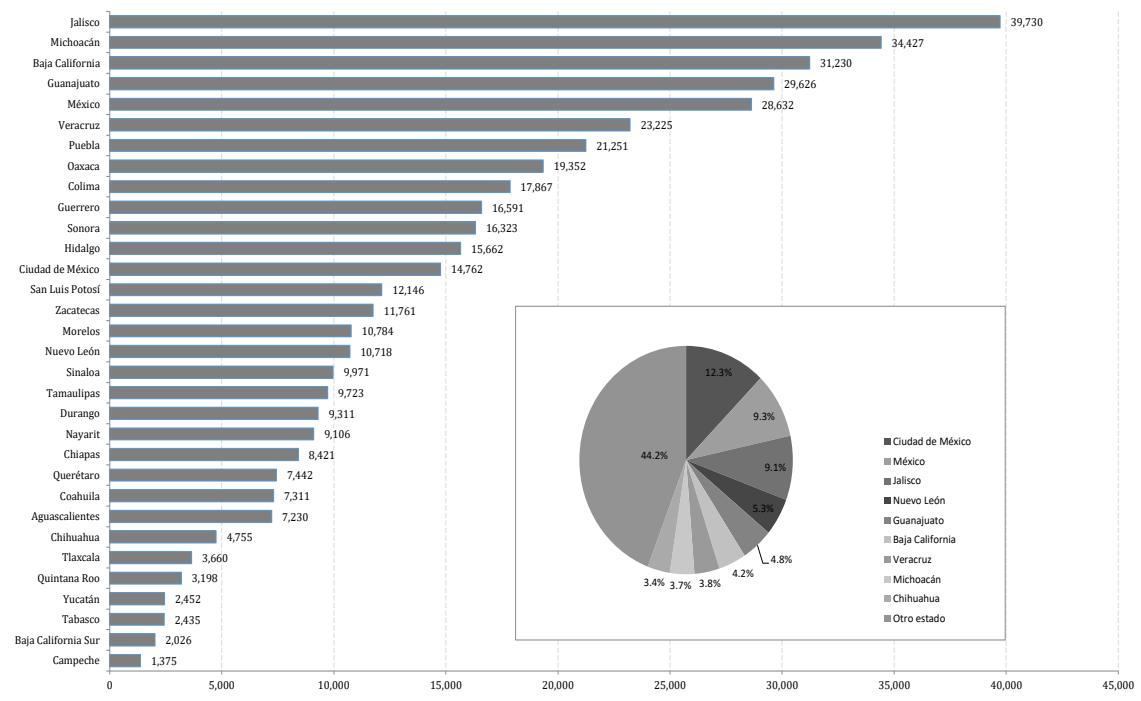

Fuente: Estimaciones de la Unidad de Política Migratoria y del Consejo Nacional de Población de la SEGOB con base en INEGI, Encuesta Intercensal, 2015; e INEGI, Censo de Población y Vivienda, 2010, muestra del 10 por ciento.

Bajo estos criterios el flujo de migrantes de retorno se estima entre 190 mil y 120 mil personas en 2014, entre 157 mil y 80 mil para 2015 y entre 163 mil y 106 mil en 2016, la estimación realizada para 2018 muestra un incremento en la tendencia de la migración de retorno mayor a la de 2017, la cual podría estar reflejando las consecuencias de la política de persecución de personas en situación migratoria irregular que lleva a cabo el actual gobierno estadounidense. 
Tabla 2. Estimación de la migración de retorno, temporalidad de estancia en Estados Unidos de América y Residencia en México, 2014-2016

\begin{tabular}{crrr}
\hline & & \multicolumn{2}{c}{ Temporalidad de estancia/permanencia } \\
\hline Estancia Estados Unidos & 6 meses & 6 meses & 1 año \\
\hline Permanencia en México & 6 meses & 1 año & 1 año \\
\hline Total 2014 & 211,189 & 190,183 & 120,436 \\
Voluntarios & 158,278 & 154,461 & 96,086 \\
Involuntarios (repatriados) & 52,911 & 35,722 & 24,350 \\
Total 2015 & 170,991 & 157,073 & 87,550 \\
Voluntarios & 127,634 & 125,562 & 65,278 \\
Involuntarios (repatriados) & 43,357 & 31,511 & 22,272 \\
Total 2016 & 176,519 & 163,402 & 106,432 \\
Voluntarios & 135,087 & 133,997 & 84,845 \\
& 41,432 & 29,405 & 21,587 \\
& Política pública & Política pública con perspectiva \\
& con perspectiva & de reintegración sin prevención. \\
& de atención con & & \\
\hline & prevención & & \\
\hline
\end{tabular}

Fuente: Estimaciones de la Unidad de Política Migratoria, la SEGOB con base en UPM, CONAPO, CONOAPRED, SRE, SEDESOL, STPS Y EL COLEF, Encuesta sobre Migración en la Frontera Norte de México (EMIF NORTE), 2014-2015; Unidad de Política Migratoria, SEGOB, a partir de la Base de datos de repatriados mexicanos.

Los escenarios en este contexto pueden ser múltiples ya que las acciones o propuestas para el manejo de la política migratoria de ese país requieren de una serie de ajustes y consensos en todo el territorio nacional. Cualquier medida, ley o decreto anti inmigratorio deberá salvar obstáculos como la resistencia de algunos gobernantes, jueces y miembros del Congreso que han marcado su desacuerdo con esa política.

Un último obstáculo, aunque no menor, es el tema del presupuesto requerido para vigilar, buscar y deportar a migrantes en situación irregular. En este escenario hipotético y considerando que actualmente se han diseñado estrategias para detener a los migrantes al interior de Estados Unidos de América con los recursos y la normatividad vigentes, el retorno estimado para 2017 ascendería a 172 mil 598 personas y alrededor de 184 mil en 2018 (véase Tabla 3). 
Tabla 3. Estimación de la migración de retorno desde Estados Unidos de América, 2014-2018

\begin{tabular}{|c|c|c|c|}
\hline \multirow[b]{2}{*}{ Año/tipo de retorno } & \multicolumn{3}{|c|}{ Personas de retorno } \\
\hline & Total & Voluntarios & Involuntario/Repatriados \\
\hline 2014 & 190,183 & 154,461 & 35,722 \\
\hline 2015 & 157,073 & 125,562 & 31,511 \\
\hline 2016 & 163,402 & 133,997 & 29,405 \\
\hline 2017 & 172,598 & 142,999 & 29,599 \\
\hline 2018 & 184,493 & 152,605 & 31,888 \\
\hline
\end{tabular}

Fuente: Estimaciones de la Unidad de Política Migratoria, la SEGOB.

\section{PERFIL SOCIODEMOGRÁFico de LA POBLACIÓN MigRANTE MEXICANA DE RETORNO PROCEDENTE DE ESTADOS UNIDOS}

Como se mencionó al inicio de este documento, parte de las estimaciones sobre el retorno de población mexicana se realizó con base en la Emif Norte, que permite cuantificar de manera continua la migración laboral desde y hacia la frontera norte de México, así como hacia y desde Estados Unidos. A continuación, se describe el perfil sociodemográfico del flujo migratorio para 2016 utilizando las estimaciones realizadas a partir de dicho instrumento, y con los criterios establecidos con anterioridad para identificar a la población migrante de retorno de 15 años o más.

\section{Migrantes de retorno voluntario desde Estados Unidos}

Las estimaciones realizadas muestran que entre la población migrante de retorno el 52 por ciento eran mujeres, porcentaje ligeramente superior al de hombres (48 por ciento), además de ser una población adulta joven, entre los 20 y 39 años (35 por ciento), con una edad promedio de 42.9 años aunque se identifica un porcentaje importante de población de 60 años o más que está de retorno en México (22 por ciento).

Una cuarta parte de estos migrantes de retorno cuenta con algún grado de secundaria, lo que en promedio se traduce en 8.8 años de escolaridad, y destaca que 17.8 por ciento cuenta con algún grado de nivel licenciatura o más escolaridad (véase Tabla 4). 
Tabla 4. Características de los migrantes de retorno voluntario que permanecieron al menos seis meses en Estados Unidos, que no piensan regresar o permanecerán en México al menos un año (procedentes de Estados Unidos vía terrestre o aérea), 2014-2016

\begin{tabular}{|c|c|c|c|}
\hline \multirow[t]{2}{*}{ Características } & \multicolumn{3}{|c|}{ Porcentajes } \\
\hline & 2014 & 2015 & 2016 \\
\hline Sexo & 100.0 & 100.0 & 100.0 \\
\hline Hombres & 46.0 & 46.6 & 48.0 \\
\hline Mujeres & 54.0 & 53.4 & 52.0 \\
\hline Grupos de edad & 100.0 & 100.0 & 100.0 \\
\hline 15 a 19 años & 7.5 & 11.5 & 9.7 \\
\hline 20 a 29 años & 23.4 & 34.4 & 20.3 \\
\hline 30 a 39 años & 16.5 & 15.1 & 14.6 \\
\hline 40 a 49 años & 16.8 & 10.6 & 14.0 \\
\hline 50 a 59 años & 14.9 & 16.6 & 19.7 \\
\hline 60 años o más & 20.9 & 11.7 & 21.7 \\
\hline Nivel educativo & 100.0 & 100.0 & 100.0 \\
\hline Sin escolaridad & 2.9 & $* 2.7$ & 6.1 \\
\hline Primaria incompleta & 14.7 & 9.4 & 13.2 \\
\hline Primaria completa & 20.3 & 15.8 & 16.6 \\
\hline Secundaria & 25.5 & 20.2 & 25.4 \\
\hline Bachillerato & 23.3 & 18.5 & 20.9 \\
\hline Licenciatura o más & 13.3 & 33.3 & 17.8 \\
\hline Condición de documentos para cruzar a EUA & 100.0 & 100.0 & 100.0 \\
\hline Con documentos & 39.0 & 52.2 & 52.8 \\
\hline Sin documentos & 61.0 & 47.8 & 47.2 \\
\hline Condición de "ayuda" para cruzar a EUA & 100 & 100.0 & 100.0 \\
\hline Con ayuda & 24.9 & 25.9 & 19.1 \\
\hline Sin ayuda & 75.1 & 74.1 & 80.9 \\
\hline Estado de mayor permanencia en EUA & 100 & 100.0 & 100.0 \\
\hline California & 39.9 & 44.9 & 50.0 \\
\hline Texas & 26.3 & 18.5 & 17.4 \\
\hline Arizona & 3.7 & 4.1 & $* 2.4$ \\
\hline Otro & 30.1 & 32.6 & 30.2 \\
\hline Condición de ocupación en EUA & 100 & 100.0 & 100.0 \\
\hline Ocupado & 22.8 & 20.3 & 25.5 \\
\hline
\end{tabular}


Tabla4. Continuación.

\begin{tabular}{|c|c|c|c|c|}
\hline \multicolumn{2}{|c|}{ No ocupado } & 77.2 & 79.7 & 74.5 \\
\hline & Ocupación en EUA & 100.0 & 100.0 & 100.0 \\
\hline & Trabajadores agropecuarios & 12.4 & 10.8 & 18.2 \\
\hline & Trabajadores industriales & 8.1 & $* 8.7$ & *12.5 \\
\hline & Trabajadores en la construcción & 19.7 & 27.3 & 20.2 \\
\hline & Comerciantes & $* 6.6$ & $* 1.0$ & $* 3.0$ \\
\hline & Trabajadores en servicios & 49.1 & 44.6 & 39.8 \\
\hline & Profesionistas, Técnicos y Personal & - & - & - \\
\hline & Administrativo & $* 4.0$ & $* 7.6$ & $* 6.3$ \\
\hline \multicolumn{2}{|c|}{ Razón de retorno a México } & 100.0 & 100.0 & 100.0 \\
\hline & Visita a familiares o de paseo & 74.2 & 74.3 & 71.9 \\
\hline & Trabajar en México & $* 0.7$ & 18.7 & 21.2 \\
\hline & Otra razón & 25.1 & 7.0 & 6.9 \\
\hline \multicolumn{2}{|c|}{ Entidad federativa a la que se dirige en México } & 100.0 & 100.0 & 100.0 \\
\hline & Jalisco & 28.6 & 37.1 & 26.8 \\
\hline & Michoacán & 13.3 & 13.3 & 9.9 \\
\hline & Ciudad de México (Distrito Federal) & 3.2 & 3.9 & 8.8 \\
\hline & Guanajuato & 5.0 & 4.7 & 5.3 \\
\hline & México & 3.5 & 5.2 & 4.8 \\
\hline & Puebla & 3.8 & 4.5 & 4.5 \\
\hline & Guerrero & 3.8 & 3.2 & 3.5 \\
\hline & Otra entidad & 38.8 & 28.1 & 36.5 \\
\hline * & \multicolumn{4}{|c|}{ Datos expandidos de muestras menores a 30 observaciones o casos. } \\
\hline
\end{tabular}

Fuente: Estimaciones del Centro de Estudios Migratorios de la UPMRIP/SEGOB con base en la Encuesta sobre Migración en la Frontera Norte de México (Emif Norte), de la UPMRIP, CONAPO, CONAPRED, SRE, SEDESOL, y EL COLEF, 2014-2016. Cuestionario de procedentes de Estados Unidos, terrestre y aeropuertos.

Cabe destacar que más de la mitad de esta población declaró haber cruzado a Estados Unidos con documentos migratorios (53 por ciento), mientras que 19.1 por ciento utilizó alguna "ayuda" pagada para cruzar, porcentaje que ha venido disminuyendo.

En lo que respecta a la residencia en Estados Unidos para esta población, la distribución geográfica no presenta novedades, pues California es el principal estado de procedencia (50 por ciento), 
seguido por Texas (17.4 por ciento); y solamente una cuarta parte de la población declaró haber trabajado durante su estancia en ese país, principalmente en ocupaciones de servicios (40 por ciento), construcción (20 por ciento) y agropecuarias (18 por ciento). En cambio, con respecto a la experiencia de retorno, una de cada cinco personas declaró que regresa a México a trabajar y este porcentaje ha ido en aumento de manera considerable: de 0.7 a 21.1 por ciento entre 2014 y 2016.

En México, las principales entidades de acogida para el retorno voluntario son aquellas de tradición migratoria como Jalisco, Michoacán, Guanajuato, Puebla y Guerrero, y se han ido sumando otras como la Ciudad de México y el Estado de México.

\section{Migrantes de retorno involuntario de Estados Unidos}

A diferencia del retorno voluntario, entre los migrante de retorno involuntario se identifica una población básicamente masculina (94 por ciento), en una edad adulta joven, de 20 a 39 años con el 69 por ciento y cuya edad promedio es de 34.2 años. En cuanto al nivel de escolaridad, el 41.2 por ciento cuenta con algún grado de secundaria, es decir, 8.1 años de educación escolar en promedio (véase Tabla 5).

Los estados de mayor permanencia en Estados Unidos para los migrantes de retorno involuntario son Texas (35 por ciento), California (21 por ciento) y Arizona (13 por ciento), estados fronterizos con México. Más de una tercera parte de esta población declaró haber trabajado en la Unión Americana. Un dato importante que debe alertar las medidas de protección dirigidas a este grupo de migrantes es que la cuarta parte declaró haber dejado hijos menores de 18 años en aquel país.

Cabe señalar que entre los migrantes de retorno involuntario detectados en los registros administrativos, entre 2015-2017 las principales ciudades de destino para los que fueron devueltos una sola vez, las principales ciudades de destino son en orden de importancia: Tijuana, Baja California; Ciudad Juárez, Chihuahua; Mexicali, Baja California; Monterrey, Nuevo León; y Reynosa, Tamaulipas. 
Tabla 5. Características de los migrantes de retorno involuntario que permanecieron al menos 6 meses en Estados Unidos, que fueron regresados por única ocasión en los últimos 12 meses y no piensan regresar a ese país (migrantes devueltos), 2014-2016

\begin{tabular}{|c|c|c|c|}
\hline \multirow{2}{*}{ Características } & \multicolumn{3}{|c|}{ Porcentajes } \\
\hline & 2014 & 2015 & 2016 \\
\hline Sexo & 100.0 & 100.0 & 100.0 \\
\hline Hombres & 92.0 & 91.2 & 94.3 \\
\hline Mujeres & 8.0 & 8.8 & $* 5.7$ \\
\hline Grupos de edad & 100.0 & 100.0 & 100.0 \\
\hline 15 a 19 años & 1.9 & 1.5 & 1.2 \\
\hline 20 a 29 años & 30.5 & 29.2 & 36.2 \\
\hline 30 a 39 años & 36.9 & 38.3 & 32.9 \\
\hline 40 a 49 años & 24.5 & 24.7 & 22.8 \\
\hline 50 a 59 años & $* 5.0$ & 5.0 & 5.2 \\
\hline 60 años o más & 1.2 & 1.5 & 1.6 \\
\hline Nivel educativo & 100.0 & 100.0 & 100.0 \\
\hline Sin escolaridad & $* 3.2$ & $* 2.4$ & $* 4.4$ \\
\hline Primaria incompleta & $* 6.8$ & 10.2 & 8.2 \\
\hline Primaria completa & 17.4 & 13.4 & 18.2 \\
\hline Secundaria & 46.5 & 47.5 & 41.2 \\
\hline Bachillerato & 23.8 & 23.6 & 26.7 \\
\hline Licenciatura o más & $* 2.2$ & $* 3.0$ & $* 1.3$ \\
\hline Condición de documentos para cruzar a EUA & 100.0 & 100.0 & 100.0 \\
\hline Con documentos & 11.1 & 9.2 & 8.2 \\
\hline Sin documentos & 88.9 & 90.8 & 91.8 \\
\hline Condición de "ayuda" para cruzar a EUA & 100.0 & 100.0 & 100.0 \\
\hline Con ayuda & 44.5 & 40.3 & 41.8 \\
\hline Sin ayuda & 55.5 & 59.7 & 58.2 \\
\hline Estado de mayor permanencia en EUA & 100.0 & 100.0 & 100.0 \\
\hline California & 28.0 & 42.3 & 34.7 \\
\hline Texas & 31.1 & 17.4 & 21.1 \\
\hline Arizona & 17.1 & 16.1 & 12.9 \\
\hline
\end{tabular}


Tabla 5. Continuación

\begin{tabular}{crrr}
\hline Otro & 23.8 & 24.2 & 31.3 \\
Condición de ocupación en EUA & 100.0 & 100.0 & 100.0 \\
Ocupado & 33.4 & 41.3 & 35.3 \\
No ocupado & 66.6 & 58.7 & 64.7 \\
Dejó hijos(as) menores de 18 años en EUA & 100.0 & 100.0 & 100.0 \\
Si dejó hijas(os) menores & 22.8 & 23.1 & 25.4 \\
No dejó hijas(os) menores & 77.2 & 76.9 & 74.6 \\
Entidad federativa a la que se dirige en México & 100.0 & 100.0 & 100.0 \\
$\quad$ Michoacán & $* 6.1$ & 5.7 & 9.2 \\
Guanajuato & $* 4.7$ & 10.4 & 7.8 \\
Baja California & 10.2 & 7.3 & 7.6 \\
Guerrero & $* 6.6$ & $* 4$ & 6.9 \\
$\quad$ Sonora & 7.8 & 6.8 & 5.1 \\
$\quad$ Otra entidad & 64.4 & 65.7 & 63.3 \\
Datos expandidos de muestras menores a 30 observaciones o casos. & \\
\hline
\end{tabular}

Fuente: Estimaciones del Centro de Estudios Migratorios de la UPMRIP/SEGOB con base en la Encuesta sobre Migración en la Frontera Norte de México (Emif Norte), de la UPMRIP, CONAPO, CONAPRED, SRE, SEDESOL, y EL COLEF, 2014-2016. Cuestionario de procedentes de Estados Unidos, terrestre y aeropuertos.

\section{Migración calificada}

La composición de los flujos migratorios es diversa y esto se hace evidente cuando se observan ciertos grupos, por ejemplo, los migrantes calificados. En el caso específico del retorno, la identificación de grupos es importante para generar condiciones óptimas para su reintegración y atención, especialmente por las diferencias que existen entre la oferta y la demanda de los mercados de trabajo mexicano y estadounidense, así como la inserción laboral de esta población en términos de expectativas y compatibilidad entre experiencia y necesidades.

Podría suponerse que los migrantes calificados cuentan con cierta ventaja para enfrentar el desempleo gracias a su talento en determinados sectores, sin embargo, esto solo sería posible en función de la demanda en el mercado al cual se insertan, es decir, si ofrece suficientes oportunidades laborales y bien compensadas.

Con base en los Censos de Población y Vivienda 1990, 2000 y 2010, así como la Encuesta Intercensal 2015, se definió aquella población 
nacida en México que hace cinco años al momento del levantamiento de la información residía en Estados Unidos y que, cuenta con nivel de estudios de licenciatura o posgrado. A partir de ello se observa que el volumen de migrantes de retorno calificados ha aumentado en las últimas décadas; el incremento más alto se alcanzó en 2010 al observarse para esa década (2000-2010) a más de 33 mil migrantes de retorno calificados, lo cual puede estar relacionado con la crisis económica mundial iniciada en Estados Unidos en 2008 y su impacto en el mercado laboral de ese país.

Para 2015 se observan 23 mil 928 migrantes de retorno calificados, los cuales representan el 6.0 por ciento del total de migrantes de retorno de ese año. Este grupo está principalmente conformado por quienes cuentan con nivel licenciatura ( 82.8 por ciento), y en una proporción menor, con especialidad o maestría (14.5 por ciento) y doctorado (2.7 por ciento), como se aprecia en la Figura 3.

Figura 2. Migrantes de retorno calificados procedentes de Estados Unidos, por quinquenio según fuente de información, 1990-2015



Fuente: Unidad de Política Migratoria, SEGOB, con base en INEGI, XI Censo General de Población y Vivienda 1990, muestra de 10 por ciento; XII Censo General de Población y Vivienda 2000, muestra de 10 por ciento; Censo de Población y Vivienda 2010, muestra de 10 por ciento y Encuesta Intercensal 2015.

En el contexto de la migración de retorno, la población calificada muestra una estructura por edad y sexo asociada a la etapa productiva ya que la edad promedio de las mujeres corresponde a 37 años y a 
38 la de hombres. Del total de población migrante calificada, 55 por ciento son hombres y 45 por ciento mujeres (véase la Figura 4).

Figura 3. Migrantes de retorno procedentes de Estados Unidos por condición de calificación, según nivel de escolaridad de la población calificada, 2015

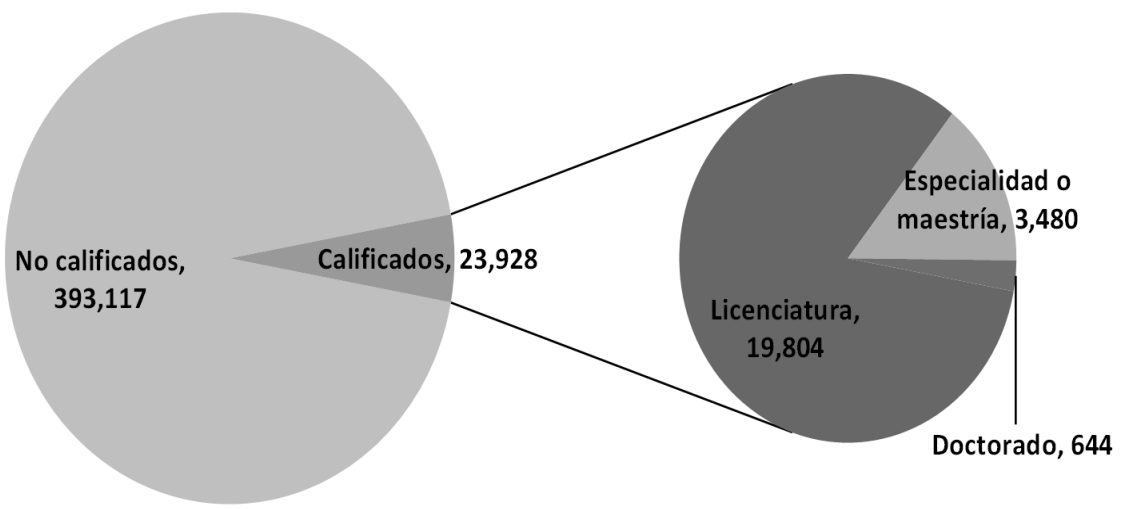

Fuente: Unidad de Política Migratoria, SEGOB, con base en INEGI, Encuesta Intercensal 2015.

Figura 4. Distribución de los migrantes de retorno calificados procedentes de Estados Unidos, según estructura por edad y sexo, 2015

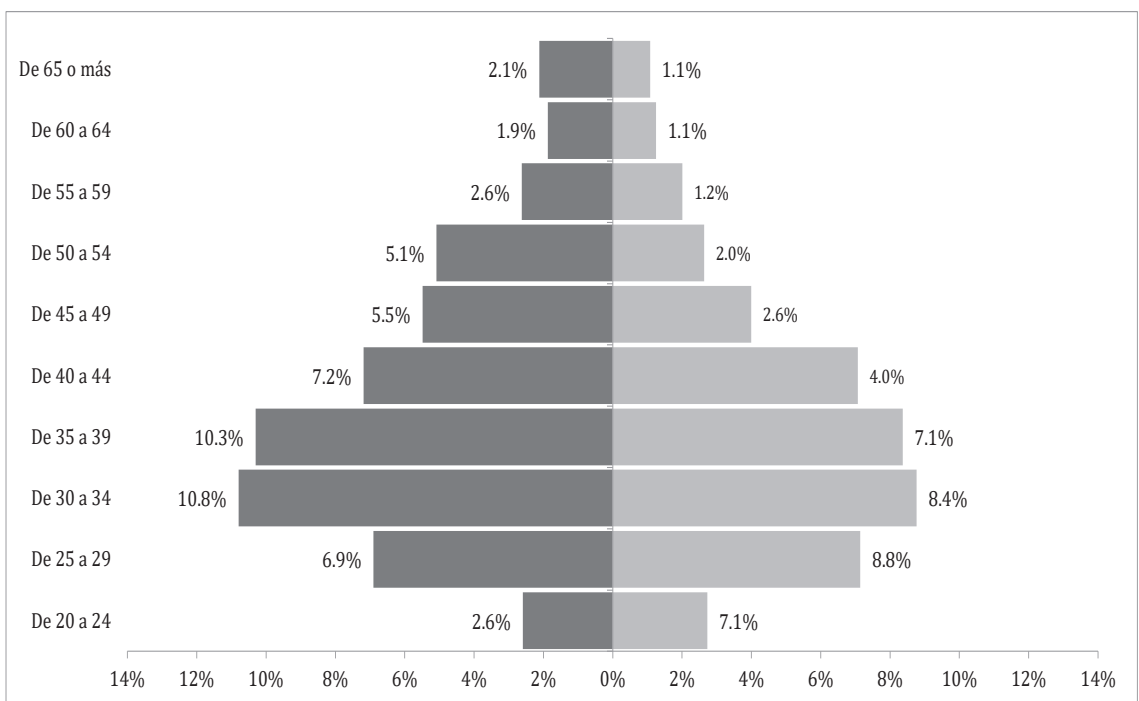

Fuente: Unidad de Política Migratoria, SEGOB, con base en INEGI, Encuesta Intercensal 2015. 
Con respecto a la inserción laboral, según estimaciones realizadas por la UPM, el 65.3 por ciento de estos migrantes trabajaba en el sector servicios y 14.5 por ciento en el comercio, mientras que, en relación a la posición en el empleo, el 69.9 por ciento se desempeñó como empleado y sólo el 19.0 por ciento trabajaba por su cuenta (UPM, 2017: 65).

Lo interesante de esto sería el aprovechamiento de las capacidades y las habilidades adquiridas en el exterior para obtener ventajas tanto para la mano de obra calificada, en términos de su reintegración a la sociedad, como del mercado receptor traducido en el conocimiento que se adquiere por la experiencia en otros contextos (know how) y que puede ser un valor agregado en el lugar de trabajo.

Lograr la conexión entre oferta y demanda en este ámbito requiere de un gran esfuerzo institucional, pero no imposible, que podría redituar grandes beneficios en el desarrollo social y económico de México. Se trata de un gran compromiso e interlocución entre las instituciones gubernamentales involucradas, como la Secretaría del Trabajo y Previsión Social, el Servicio Nacional de Empleo, el Consejo Nacional de Normalización y Certificación de Competencias Laborales, la Secretaría de Bienestar, la Secretaría de Economía, entre las principales, bajo la coordinación de las Secretarías de Relaciones Exteriores y de Gobernación como responsables de implementar la política migratoria en el país.

\section{COMENTARIOS FINALES}

En este documento presentamos propuestas que abonan a la medición y el entendimiento de la migración de retorno. A partir de la literatura disponible, se retoma una conceptualización para abordar este tema de investigación y posteriormente se establecen criterios para su medición en función de ciertas hipótesis, como: a) la temporalidad de estancia en Estados Unidos; b) la temporalidad de permanencia en México, y c) las veces que fue devuelto por una autoridad migratoria a México, y se conformaron definiciones intermedias del retorno según las fuentes de información disponibles: Censos y Encuesta Intercensal, Encuesta sobre Migración en la Frontera Norte de México (Emif Norte) y Estadísticas Migratorias.

En función de lo anterior, analizamos los distintos escenarios que pueden plantearse para lograr una mayor precisión de las estimaciones de la migración de retorno en virtud de ciertas hipótesis. Sin embargo, 
consideramos que más allá de las aproximaciones técnicas para su medición el reto que tenemos frente a este tema es la necesidad de contar con una política adecuada para la reintegración a la sociedad de las mexicanas y mexicanos que regresan a México, para quienes es indispensable garantizar como piso mínimo el acceso a los bienes y servicios indispensables para una vida digna. Especialmente, en un nivel de análisis más profundo es indispensable hacer frente a los retos que impone la reunificación de las familias.

Otro planteamiento de este trabajo consistió en la necesidad de contar con políticas gubernamentales de cara a la migración de retorno, al menos, desde dos perspectivas: una, de reintegración plena de las y los connacionales que retornan a México de manera voluntaria o involuntaria; y, la segunda, en aras de promover alternativas a la migración aminorando los motivos que la desencadenan como el desarrollo económico del país a nivel regional, la generación de oportunidades y bienestar a la población mexicana.

Uno de los grandes obstáculos que hemos identificado para el buen funcionamiento de una política en la materia, es la incompatibilidad de las acciones puestas en marcha en los tres órdenes de gobierno, lo que ha repercutido directamente en la generación de condiciones de vulnerabilidad para las personas migrantes, ya que las disposiciones del gobierno federal no siempre son adoptadas en las entidades federativas y en los ámbitos de las localidades más desagregadas.

Como parte de nuestro análisis nos enfocamos en una de las dimensiones de la migración de retorno que, a nuestro parecer, requiere de medidas de atención concretas que pueden repercutir en un beneficio para el mercado laboral de nuestro país. Nos referimos a la migración calificada, considerando que es necesario propiciar su incorporación al mercado laboral mediante políticas asertivas de coordinación entre los sectores público y privado, así como de estrategias que faciliten su incorporación considerando las habilidades con las que cuenta esta mano de obra a fin de aprovechar su potencial, lo que sin duda, tendría beneficios en los lugares de acogida. En este marco es necesario considerar la certificación de las competencias y habilidades como parte de los procesos de integración.

Hay que considerar que una parte de la migración de retorno tiene su origen en la voluntad de las personas migrantes, quienes en muchos casos regresan con recursos económicos suficientes para llevar a cabo 
un negocio por su cuenta y lo que requieren es apoyo por parte de los gobiernos y el acceso a programas sociales para su impulso.

Finalmente, consideramos necesario generar en México condiciones de bienestary desarrollo para toda la población, en igualdady al margen de su estatus migratorio. En este sentido, la política de Estado debe ser incluyente, transversal a todos los órdenes de gobierno y diferenciada según los diversos perfiles de la población que regresa, y sobre todo, incorporar las dimensiones de acompañamiento y seguimiento a estos procesos.

\section{REFERENCIAS BIBLIOGRÁFICAS}

Department of Homeland Security (blog). (2015). “2015 Yearbook of Immigration Statistics", Estados Unidos: Department of Homeland Security Office of Immigration Statistics. Consultado el 8 de junio de 2017 en https://www.dhs.gov/immigration-statistics/yearbook/2015

Instituto Nacional de Estadística y Geografía (INEGI). (2014). Encuesta Nacional de la Dinámica Demográfica.

Instituto Nacional de Estadística y Geografía (INEGI). (2015). Encuesta Intercensal, 2015.

Instituto Nacional de Estadística y Geografía (INEGI). (2010). Censo de Población y Vivienda, muestra del 10 por ciento.

Martin, Susan; Sanjula Weerasinghe y Abbie Taylor. (2014). “ $¿$ What is crisis migration?", en Migraciones Forzadas, España, Instituto Interuniversitario de Desarrollo Social y Paz, Universidad de Alicante, núm. 45, marzo, pp. 5-9.

Organización Internacional para las Migraciones. (2006). "Glosario sobre Migración", Serie de Derecho Internacional sobre Migración, núm. 7, Ginebra, Suiza.

The White House [blog]. (2017). Executive Order: Enchancing Public Safety in the Interior of the United States, Washington. Consultado el 25 de enero de 2017 en https://www.whitehouse.gov/the-press-office/2017/01/25/ presidential-executive-order-enchancing-public-safety-interior-united

Unidad de Política Migratoria (UPM). (2017). “Prontuario sobre migración mexicana de retorno", México. SEGOB, 108 p.

Unidad de Política Migratoria. (2014). SEGOB Base de datos de repatriados mexicanos 2014.

Unidad de Política Migratoria. (20015).SEGOB Base de datos de repatriados mexicanos 2015.

Unidad de Política Migratoria. (2016). SEGOB Base de datos de repatriados mexicanos 2016. 
UPM, CONAPO, CONAPRED, SRE, SEDESOL, STPS y EL COLEF. (2014). Encuesta sobre Migración en la Frontera Norte de México (EMIF NORTE) 2014.

UPM, CONAPO, CONAPRED, SRE, SEDESOL, STPS y EL COLEF. (2015). Encuesta sobre Migración en la Frontera Norte de México (EMIF NORTE) 2015.

UPM, CONAPO, CONAPRED, SRE, SEDESOL, STPS y EL COLEF. (2016). Encuesta sobre Migración en la Frontera Norte de México (EMIF NORTE) 2016.

\section{RESUMEN CURRICULAR DE LAS AUTORAS}

\section{Graciela Martínez Caballero}

Doctora en Geografía por la Universidad Nacional Autónoma de México, maestra en Demografía por El Colegio de la Frontera Norte y egresada de la Licenciatura en Sociología en la UNAM. Desde septiembre de 2009 es Directora de Estadística del Centro de Estudios Migratorios de la Unidad de Política Migratoria, Registro e Identidad de Personas. Ha publicado artículos especializados en temas migratorios, el último es Trazando rutas de la migración de tránsito irregular o no documentada por México. Es miembro de los Comités Técnicos Especializados de Estadística Económicas del Sector Turismo y Dinámica Demografía del SNIEG-INEGI.

Dirección electrónica: gmartinezc@segob.gob.mx

\section{Mónica Martínez de la Peña}

Es doctora en Estudios de Población, maestra en Demografía y licenciada en Sociología. Cuenta con trece años de experiencia en el servicio público. Ha colaborado en el área de estadística del Instituto Nacional de las Mujeres; en la Comisión de Derechos Humanos del Distrito Federal, dirigió primero el Centro de Investigación Aplicada y posteriormente la Coordinación del Servicio Profesional. Actualmente es Subdirectora de Investigación en la Unidad de Política Migratoria, Registro e Identidad de Personas, de la Secretaría de Gobernación, en donde colabora en procesos de investigación que contribuyan al entendimiento del fenómeno migratorio en el país y sus retos.

Dirección electrónica: momartinezp@segob.gob.mx 